\title{
Estimation of Activity Coefficients for binary mixture VLE Data using MATLAB
}

\author{
Tarun Jain $^{1}$, Parminder Singh ${ }^{2}$ \\ Student, Department of Chemical Engineering, Thapar University Patiala, India ${ }^{1}$ \\ Assistant Professor, Department of Chemical Engineering, Thapar University Patiala, India ${ }^{2}$
}

\begin{abstract}
Vapor- liquid equilibrium data for three strongly associated binary systems was taken for estimation of parameters of four different thermodynamic models by developing a general code using optimization techniques in MATLAB software. Isobaric vapor-liquid equilibrium measurements are considered in this work for three binary systems methanol-ethanoic acid, methanol-water and water-ethanoic acid at $101.325 \mathrm{kpa}$. None of the systems form an azeotrope. The activity coefficient has been calculated taking into consideration the non-ideality of the mixture. The experimental T, x, y data are used to estimate nonrandom two-liquid (NRTL), Wilson, Margules and VanLaar model parameters, and these parameters in turn are used to calculate vapor phase compositions. The activity coefficients of the solution were correlated with NRTL, Wilson, Margules, and VanLaar models through fitting by least-squares method. The VLE data of binary system were well predicted from these binary interaction parameters of NRTL, Wilson, Margules, and VanLaar model parameters without any additional adjustment to build the thermodynamic model of VLE for binary system and obtain the vapor-pressure compositions and the calculated bubble points.
\end{abstract}

Keywords: Binary mixture, Vapor liquid equilibrium, Wilson, Margules, VanLaar, NRTL.

\section{INTRODUCTION}

Mass transfer operations such as distillation are extensively used for separation or extraction of large number of liquid mixtures. Due to this, the knowledge of Vapor liquid equilibrium data is of great importance when it comes to accurate designing of required equipment. Nonideality plays a very important role in industrial processes and equipment design calculations.

When VLE data is generated and phases are analyzed using samples. While sampling the compositions of both liquid and gaseous phase change and accordingly behavior of system also changes. This decreases the possibility of highly accurate VLE data.

This fact points towards the necessity for much analytical work which leads to enhance an interest in exploring new methods for determination of equilibrium data that do not involve sampling and analysis of vapor phase components. Several methods have been suggested for determination of accurate data. Van Ness and coworkers classified these methods in two categories, direct and indirect methods [1]. Direct method contains calculation of vapor compositions by integration of coexistence equation which was derived from Gibbs-Duhem equation [2]. Indirect methods involve ascertaining which of the selected solution equations to the Gibbs-Duhem equation which lead to best fit to the experimental data, and determination of parametric values producing the best fit. This paper reports novel correlation and prediction of VLE data for these isobaric binary systems at $101.25 \mathrm{kpa}$. Non ideality of mixture was corrected by the calculation of its activity coefficient, which was obtained based on NRTL, Wilson, Margules and VanLaar models as function of T,x,y through nonlinear fit of least squares method.

\section{RESULTS AND DISCUSSION}

2.1 Calculation of vapor-phase Mole Fraction y for Binary Systems from Model

There are several methods concerning the correlation and predicting VLE data. VLE data can also be predicted and correlated using model-free approach. It was suggested by Wisniak's group that novel model-free computation techniques and limiting conditions have been applied for azeotropic systems [3]. But Segura and coworkers stated that model-free approach dealt with VLE data in application of ternary systems $[4,5]$. In this paper, for all three binary systems, activity coefficients were correlated with Wilson [6], Margules [7], NRTL [8] and Van-Laar [9] equations. The Interaction parameters were computed by minimizing the objective function using fitting by leastsquares method.

2.2 Equations and models used for calculations are-

1. Wilson Model

One limitation of Wilson's equation is that it is unable to model liquid-liquid equilibria, but it is reasonably accurate for modeling the liquid phase when correlating the vaporliquid equilibria of many mixtures. Wilson equation should be used only for liquid systems that are completely miscible or else for those limited regions of partially limited systems where only one liquid phase is present.

$$
\begin{aligned}
& \ln \gamma_{1}=-\ln \left(\mathrm{x}_{1}+\Lambda_{12} \mathrm{x}_{2}\right)+\mathrm{x}_{2}\left(\frac{\Lambda 12}{\mathrm{x} 1+\Lambda 12 \mathrm{x} 2}-\frac{\Lambda 21}{\mathrm{x} 2+\Lambda 21 \mathrm{x} 1}\right) \\
& \ln \gamma_{2}=-\ln \left(\mathrm{x}_{2}+\Lambda_{21} \mathrm{x}_{1}\right)+\mathrm{x}_{1}\left(\frac{\Lambda 12}{\mathrm{x} 1+\Lambda 12 \times 2}-\frac{\Lambda 21}{\mathrm{x} 2+\Lambda 21 \times 1}\right)
\end{aligned}
$$

\section{Van-Laar Model}

The Van-Laar equation was derived from Van der Waals equation. The original van der Waals parameters didn't 
Vol. 3, Issue 10, October 2016

give good description of vapor-liquid phase equilibria, concentration around a molecule is different from the bulk which forced the user to fit the parameters to experimental concentration.

results. Because of this, the model lost the connection to molecular properties, and therefore it has to be regarded as an empirical model to correlate experimental results.

$$
\begin{aligned}
& \ln \gamma_{1}=A_{12}\left(\frac{A 21 \times 2}{A 12 \times 1+A 21 \times 2}\right)^{2} \\
& \ln \gamma_{1}=A_{21}\left(\frac{A 21 \times 1}{A 12 \times 1+A 21 \times 2}\right)^{2}
\end{aligned}
$$

\section{Margules Model}

The Margules activity model is a simple thermodynamic model for the excess Gibbs free energy of a liquid mixture introduced in 1895 by Max Margules. In chemical engineering the Margules Gibbs free energy model for liquid mixtures is better known as the Margules activity or activity coefficient model. Although the model is old it has the characteristic feature to describe extrema in the activity coefficient which other models cannot.

$$
\begin{aligned}
& \ln \gamma_{1}=\left[\mathrm{A}_{12}+2\left(\mathrm{~A}_{21}-\mathrm{A}_{12}\right) \mathrm{x}_{1}\right] \mathrm{x}_{2}{ }^{2} \\
& \ln \gamma_{2}=\left[\mathrm{A}_{21}+2\left(\mathrm{~A}_{12}-\mathrm{A}_{21}\right) \mathrm{x}_{2}\right] \mathrm{x}_{1}{ }^{2}
\end{aligned}
$$

\section{NRTL (Non-Random Two-Liquid) Model}

The Non-random two-liquid model is an activity coefficient model that correlates the activity coefficients of a compound $i$ with its mole fractions in the liquid phase concerned. It is frequently applied in the field of chemical engineering to calculate phase equilibria. The concept of NRTL is based on the hypothesis of Wilson that the local

$$
\begin{gathered}
\ln \gamma_{1}=\mathrm{x}_{2}{ }^{2}\left[\tau_{21}\left(\frac{\mathrm{G} 21}{\mathrm{x} 1+\mathrm{x} 2 \mathrm{G} 21}\right)^{2}+\tau_{12} \frac{\mathrm{G} 12}{(\mathrm{x} 2+\mathrm{x} 1 \mathrm{G} 12) 2}\right] \\
\ln \gamma_{2}=\mathrm{x}_{1}{ }^{2}\left[\tau_{12}\left(\frac{\mathrm{G} 12}{\mathrm{x} 2+\mathrm{x} 1 \mathrm{G} 12}\right)^{2}+\tau_{21} \frac{\mathrm{G} 21}{(\mathrm{x} 1+\mathrm{x} 2 \mathrm{G} 21) 2}\right] \\
\operatorname{lnG}_{12}=-\alpha_{12} \tau_{12} \\
\operatorname{lnG}_{21}=-\alpha_{21} \tau_{21}
\end{gathered}
$$

Objective function in our work was taken as -

$$
\text { Objective function }=\sum_{i=1}^{N}\left(\frac{\gamma_{i, \text { cal }}-\gamma_{i, \text { exp }}}{2}\right)^{2}
$$

Where $\mathrm{N}$ is the number of experimental points, $\gamma \mathrm{i}$,calc , $\gamma$ i,exp are activity coefficients of component $\mathrm{i}$ calculated and experimental values from measured data, respectively. The method of least squares is a standard approach in regression analysis to the approximate solution of overdetermined systems, i.e., sets of equations in which there are more equations than unknowns. "Least squares" means that the overall solution minimizes the (6um of the squares of the errors made in the results of every single equation. The most important application is in data fitting. The best fit in the least-squares sense minimizes the sum of squared residuals, a residual being the difference between an observed value and the fitted value provided by a model.

Table 1: Antoine coefficients of the compounds

\begin{tabular}{|c|c|c|c|}
\hline Compound & Ai & Bi & Ci \\
\hline Methanol & 7.19736 & 1574.99 & -34.29 \\
\hline Water & 7.07404 & 1657.46 & -46.13 \\
\hline Ethanoic acid & 6.42452 & 1479.02 & -56.34 \\
\hline
\end{tabular}

Table 2: Correlation parameters of activity coefficient

\begin{tabular}{|c|c|c|c|c|}
\hline Equation & Parameters & Methanol(1)+water(2) & $\begin{array}{c}\text { Methanol(1)+ Ethanoic } \\
\text { acid (2) }\end{array}$ & $\begin{array}{c}\text { Water(1)+Ethanoic acid } \\
\text { (2) }\end{array}$ \\
\hline Van-Laar & $\mathrm{A}_{12}$ & 0.8407 & - & 0.2777 \\
& $\mathrm{~A}_{21}$ & 0.5386 & - & 0.4607 \\
& $\mathrm{Fmin}$ & 0.0075 & - & 0.0421 \\
\hline Margules & $\Lambda_{12}$ & 0.8256 & -0.335 & 0.2416 \\
& $\Lambda_{21}$ & 0.4791 & 0.0219 & 0.4632 \\
& $\mathrm{Fmin}$ & 0.0080 & 0.0599 & 0.0360 \\
\hline Wilson & $\mathrm{A}_{12}$ & 0.4139 & 0.5307 & 1.2795 \\
& $\mathrm{~A}_{21}$ & 1.0354 & 1.8843 & 0.4759 \\
& $\mathrm{Fmin}$ & 0.0074 & 0.0784 & 0.0423 \\
\hline NRTL & $\mathrm{G}_{12}$ & -0.2831 & 2.7759 & 1.0233 \\
& $\mathrm{G}_{21}$ & 1.1457 & -1.5237 & -0.4846 \\
& $\mathrm{Fmin}$ & 0.0075 & 0.0390 & 0.0405 \\
\hline
\end{tabular}

Table 3: Experimental data for Methanol (1) + Water (2) System at 101.25 kpa

\begin{tabular}{|c|c|c|c|c|}
\hline $\mathrm{x}_{1}$ & $\mathrm{y}_{1}$ & $\mathrm{~T}_{\exp }$ & $\gamma_{1 \exp }$ & $\gamma_{2 \exp }$ \\
\hline 0.0484 & 0.2704 & 365.54 & 2.1436 & 1.0034 \\
\hline 0.0539 & 0.3009 & 364.88 & 2.1147 & 1.0042 \\
\hline
\end{tabular}


International Advanced Research Journal in Science, Engineering and Technology ISO 3297:2007 Certified

Vol. 3, Issue 10, October 2016

\begin{tabular}{|l|l|l|l|l|}
\hline 0.0607 & 0.3213 & 364.12 & 2.0799 & 1.0053 \\
\hline 0.0696 & 0.3492 & 363.19 & 2.0358 & 1.0069 \\
\hline 0.0806 & 0.3866 & 362.13 & 1.9835 & 1.0092 \\
\hline 0.0911 & 0.4126 & 361.21 & 1.9360 & 1.0117 \\
\hline 0.1056 & 0.4492 & 360.05 & 1.8739 & 1.0155 \\
\hline 0.1197 & 0.4762 & 359.04 & 1.8172 & 1.0197 \\
\hline 0.1354 & 0.4885 & 358.02 & 1.7584 & 1.0248 \\
\hline 0.1436 & 0.5047 & 357.53 & 1.7293 & 1.0277 \\
\hline 0.1586 & 0.5323 & 356.70 & 1.6788 & 1.0334 \\
\hline 0.1815 & 0.5677 & 355.56 & 1.6083 & 1.0428 \\
\hline 0.2092 & 0.5969 & 354.35 & 1.5325 & 1.0555 \\
\hline 0.2202 & 0.6031 & 353.91 & 1.5049 & 1.0609 \\
\hline 0.2380 & 0.6237 & 353.24 & 1.4633 & 1.0701 \\
\hline 0.2648 & 0.6519 & 352.32 & 1.4066 & 1.0847 \\
\hline 0.2918 & 0.6681 & 351.48 & 1.3561 & 1.1005 \\
\hline 0.3166 & 0.6923 & 350.76 & 1.3148 & 1.1157 \\
\hline 0.3442 & 0.7197 & 350.02 & 1.2740 & 1.1336 \\
\hline 0.4015 & 0.7431 & 348.61 & 1.2038 & 1.1730 \\
\hline 0.4282 & 0.7443 & 348.00 & 1.1767 & 1.1924 \\
\hline 0.4644 & 0.7613 & 347.21 & 1.1449 & 1.2194 \\
\hline 0.5240 & 0.7860 & 345.98 & 1.1026 & 1.2656 \\
\hline 0.6091 & 0.8231 & 344.33 & 1.0595 & 1.3338 \\
\hline 0.6899 & 0.8633 & 342.85 & 1.0325 & 1.3995 \\
\hline 0.8213 & 0.9221 & 340.56 & 1.0086 & 1.5038 \\
\hline & & & & \\
\hline
\end{tabular}

Table 4: Experimental data for Methanol (1) + Ethanoic acid (2) System at 101.25 kpa

\begin{tabular}{|c|c|c|c|c|}
\hline $\mathbf{x}_{\mathbf{1}}$ & $\mathbf{y}_{\mathbf{1}}$ & $\mathbf{T}_{\mathbf{e x p}}$ & $\boldsymbol{\gamma}_{\text {1exp }}$ & $\boldsymbol{\gamma}_{\text {2exp }}$ \\
\hline 0.0359 & 0.1574 & 386.23 & 0.9388 & 0.9999 \\
\hline 0.0410 & 0.1664 & 385.56 & 0.9393 & 0.9999 \\
\hline 0.0648 & 0.2719 & 382.68 & 0.9419 & 0.9997 \\
\hline 0.0741 & 0.3133 & 381.90 & 0.9428 & 0.9996 \\
\hline 0.0859 & 0.3333 & 380.60 & 0.9441 & 0.9995 \\
\hline 0.1040 & 0.3830 & 379.06 & 0.9460 & 0.9992 \\
\hline 0.1280 & 0.4602 & 377.35 & 0.9485 & 0.9988 \\
\hline 0.1629 & 0.5213 & 374.70 & 0.9521 & 0.9981 \\
\hline 0.1919 & 0.5873 & 372.43 & 0.9551 & 0.9973 \\
\hline 0.2510 & 0.6495 & 368.44 & 0.9609 & 0.9954 \\
\hline 0.3391 & 0.7538 & 363.56 & 0.9692 & 0.9916 \\
\hline 0.4401 & 0.8232 & 357.71 & 0.9776 & 0.9857 \\
\hline 0.5829 & 0.8999 & 350.75 & 0.9874 & 0.9748 \\
\hline 0.7050 & 0.9389 & 346.39 & 0.9936 & 0.9633 \\
\hline 0.7509 & 0.9533 & 344.06 & 0.9955 & 0.9583 \\
\hline 0.8109 & 0.9693 & 342.35 & 0.9974 & 0.9515 \\
\hline 0.8711 & 0.9766 & 340.75 & 0.9988 & 0.9442 \\
\hline 0.9161 & 0.9850 & 340.00 & 0.9995 & 0.9384 \\
\hline
\end{tabular}

Table 5: Experimental data for Water (1) + Ethanoic acid (2) System at $101.25 \mathrm{kpa}$

\begin{tabular}{|c|c|c|c|c|}
\hline $\mathbf{x}_{\mathbf{1}}$ & $\mathbf{y}_{\mathbf{1}}$ & $\mathbf{T}_{\mathbf{e x p}}$ & $\boldsymbol{\gamma}_{\mathbf{1} \exp }$ & $\boldsymbol{\gamma}_{\mathbf{2 e x p}}$ \\
\hline 0.0665 & 0.1140 & 387.22 & 1.4161 & 1.0025 \\
\hline 0.0749 & 0.1288 & 387.00 & 1.4046 & 1.0031 \\
\hline 0.0855 & 0.1603 & 386.48 & 1.3903 & 1.0040 \\
\hline 0.0995 & 0.1938 & 385.86 & 1.3117 & 1.0054 \\
\hline 0.1507 & 0.2802 & 384.16 & 1.2888 & 1.0121 \\
\hline 0.1722 & 0.3155 & 383.44 & 1.2684 & 1.0156 \\
\hline 0.2168 & 0.3676 & 382.39 & 1.2171 & 1.0242 \\
\hline
\end{tabular}


International Advanced Research Journal in Science, Engineering and Technology

ISO 3297:2007 Certified

Vol. 3, Issue 10, October 2016

\begin{tabular}{|l|l|l|l|l|}
\hline 0.2502 & 0.4101 & 381.53 & 1.1838 & 1.0316 \\
\hline 0.2936 & 0.4407 & 380.94 & 1.1589 & 1.0425 \\
\hline 0.3303 & 0.4815 & 380.20 & 1.1318 & 1.0528 \\
\hline 0.3756 & 0.5253 & 379.40 & 1.0965 & 1.0666 \\
\hline 0.4465 & 0.5890 & 378.27 & 1.0768 & 1.0908 \\
\hline 0.4946 & 0.6274 & 377.61 & 1.0543 & 1.1087 \\
\hline 0.5615 & 0.6777 & 376.80 & 1.0427 & 1.1355 \\
\hline 0.6036 & 0.7137 & 376.24 & 1.0315 & 1.1533 \\
\hline 0.6517 & 0.7546 & 375.59 & 1.0213 & 1.1746 \\
\hline 0.7067 & 0.7849 & 375.11 & 1.0217 & 1.1998 \\
\hline 0.7045 & 0.7848 & 375.11 & 1.0148 & 1.1988 \\
\hline 0.7507 & 0.8173 & 374.71 & 1.0091 & 1.2207 \\
\hline 0.8008 & 0.8501 & 374.31 & 1.0035 & 1.2451 \\
\hline 0.8723 & 0.9011 & 373.80 & 1.0016 & 1.2809 \\
\hline 0.9116 & 0.9315 & 373.54 & 1.0004 & 1.3009 \\
\hline
\end{tabular}

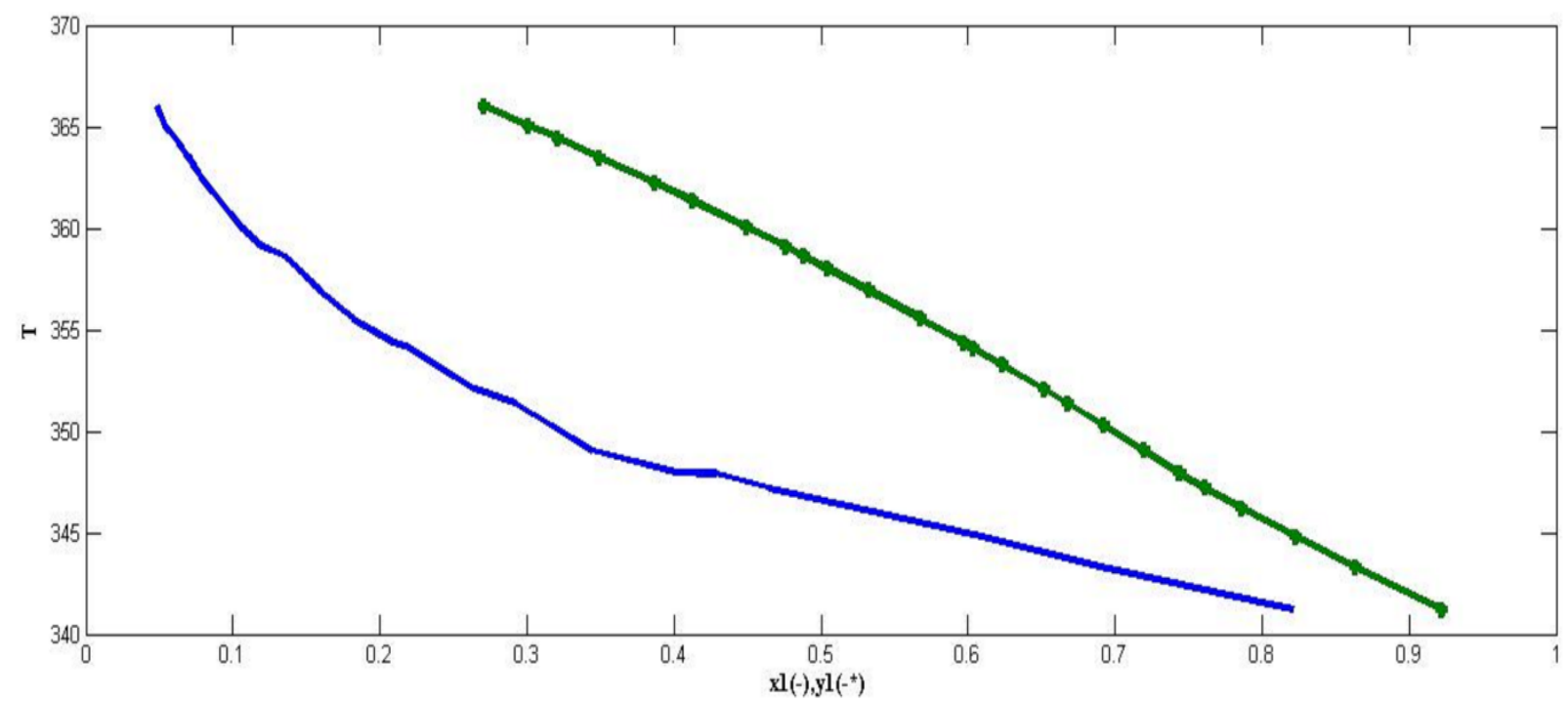

Figure 1- T-xy diagram of Methanol (1) +Water(2) System at $101.25 \mathrm{kpa}$

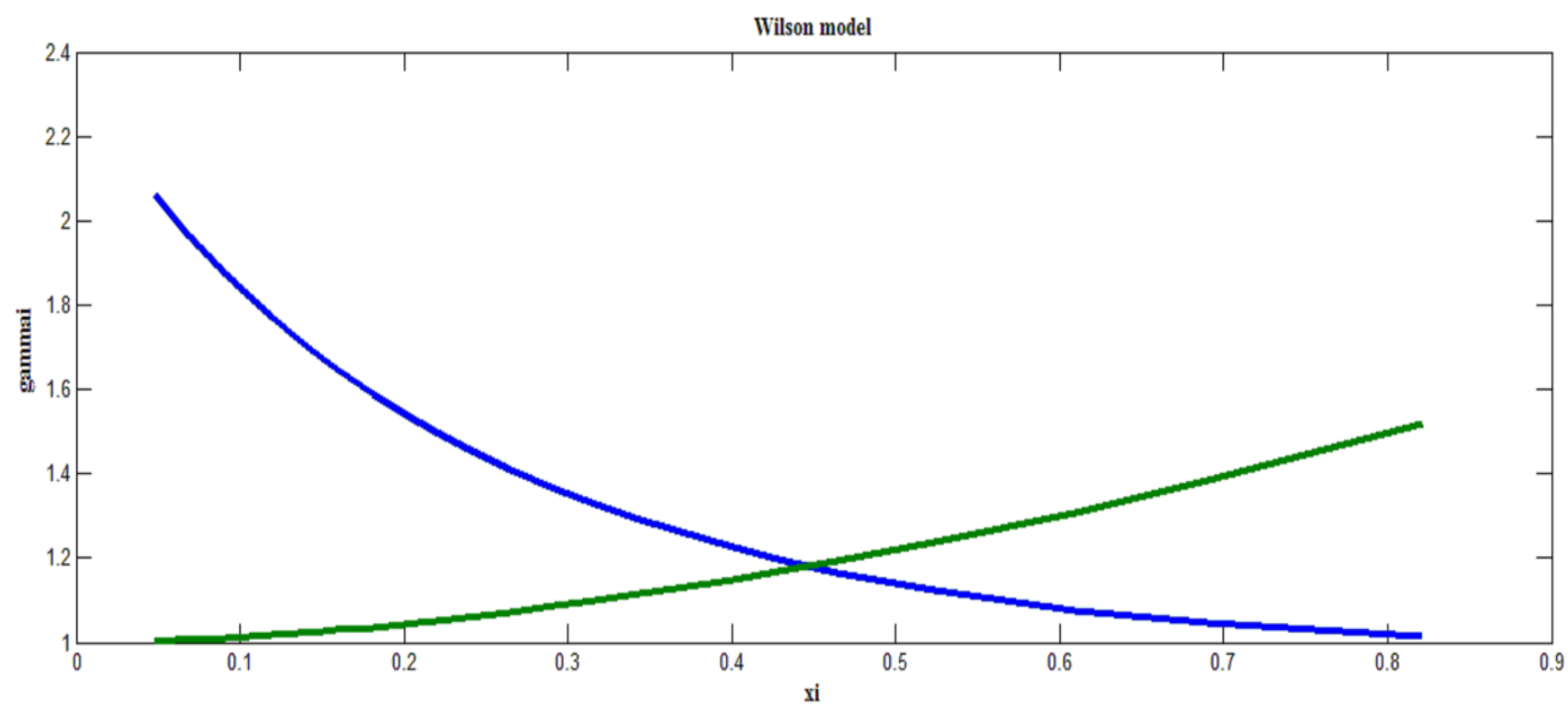

Figure 2- Plot of $\gamma_{1}$ and $\gamma_{2}$ vs $\mathrm{x}_{1}$ of Methanol(1)+Water(2) System at $101.25 \mathrm{kpa}$ 


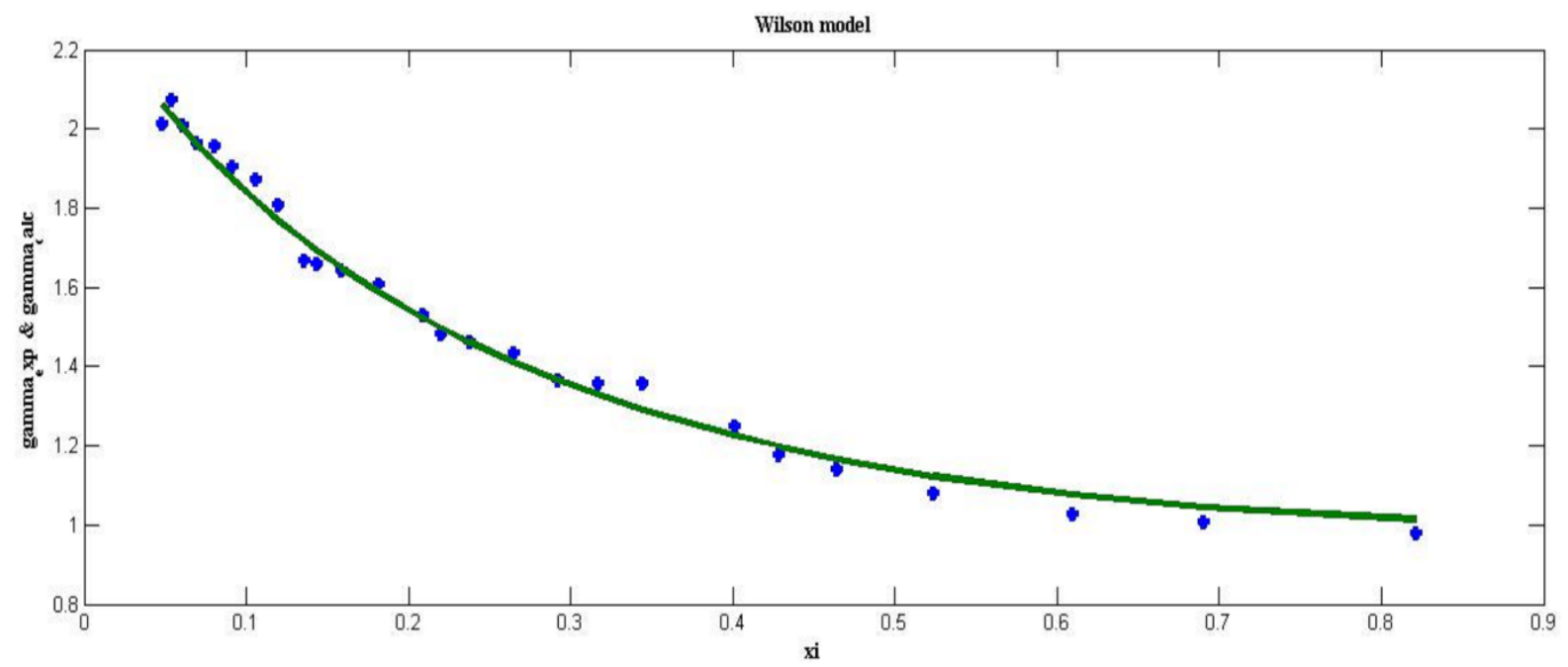

Figure 3- $\gamma_{\text {calc }}$ and $\gamma_{\text {exp }}$ vs $\mathrm{x}_{1}$ of Methanol(1)+Water(2) System at $101.25 \mathrm{kpa}$

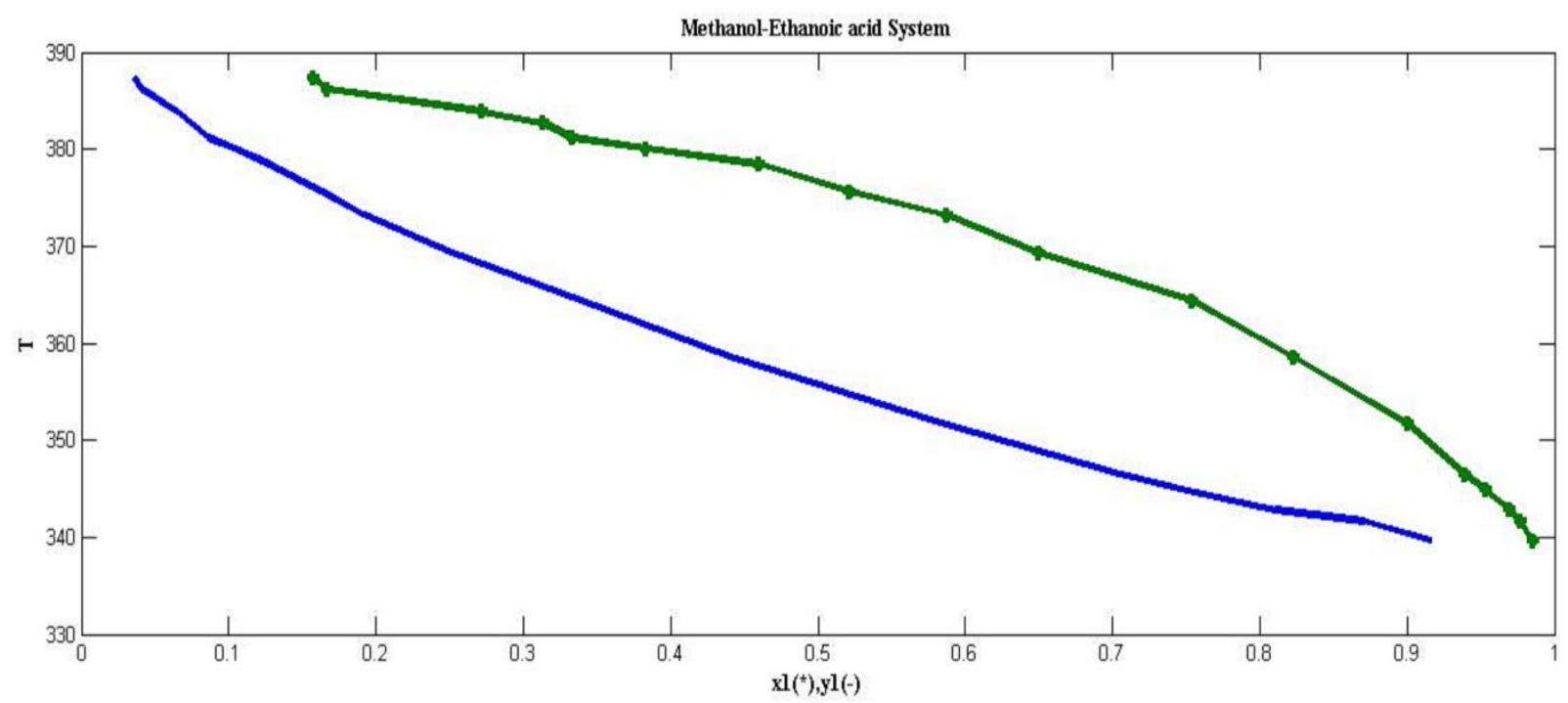

Figure 4- T-xy diagram of Methanol(1)+Ethanoic acid(2) System at $101.25 \mathrm{kpa}$

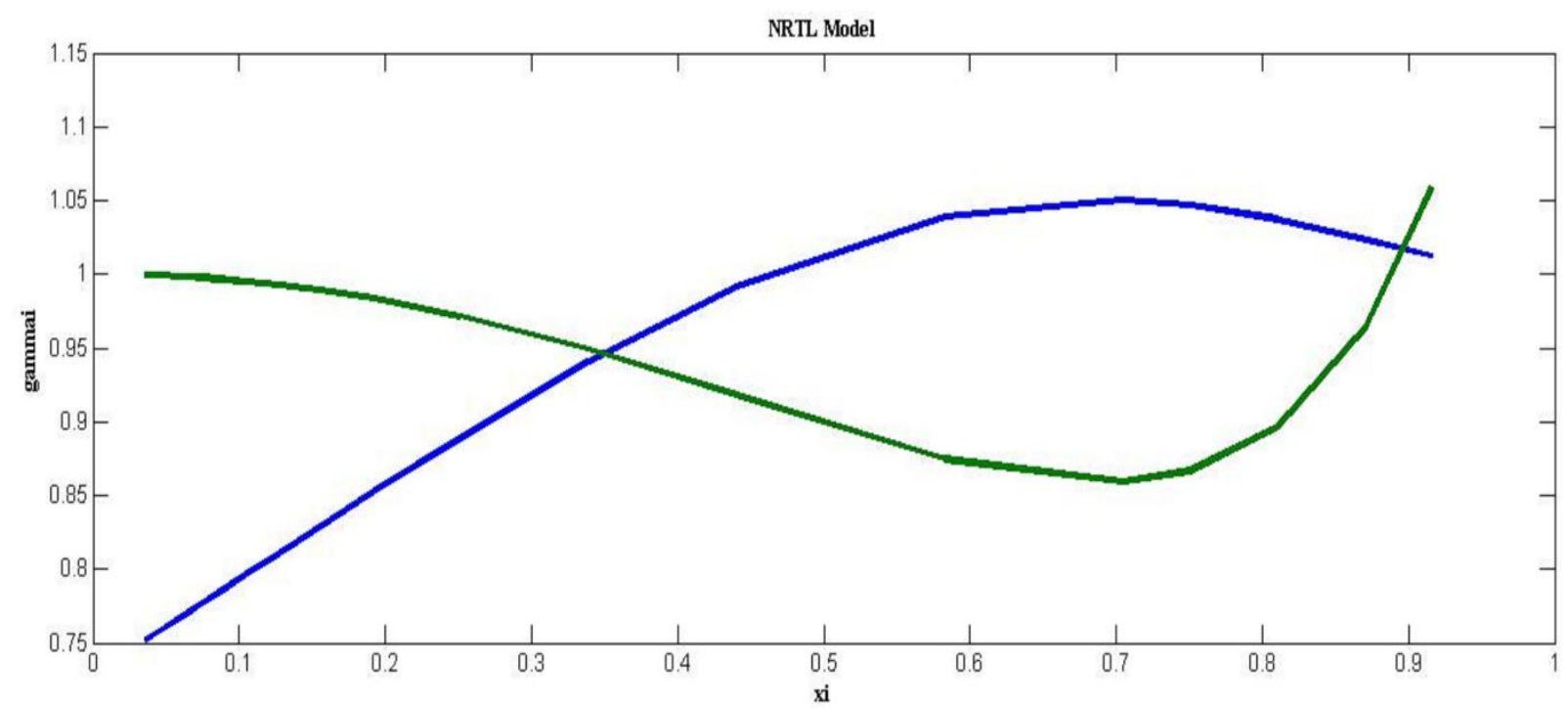

Figure 5- Plot of $\gamma_{1}$ and $\gamma_{2}$ vs $\mathrm{x}_{1}$ of Methanol(1)+Ethanoic acid(2) System at $101.25 \mathrm{kpa}$ 


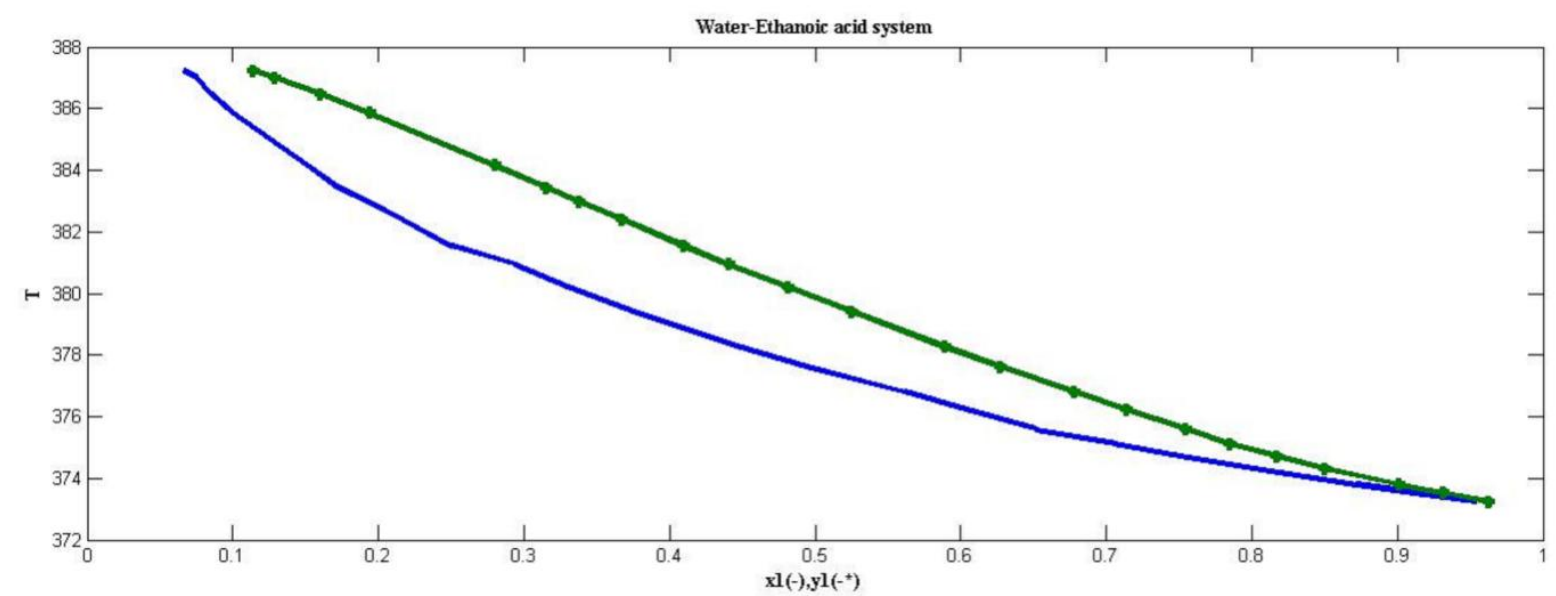

Figure 6- T-xy diagram of Water(1)+Ethanoic acid(2) System at $101.25 \mathrm{kpa}$

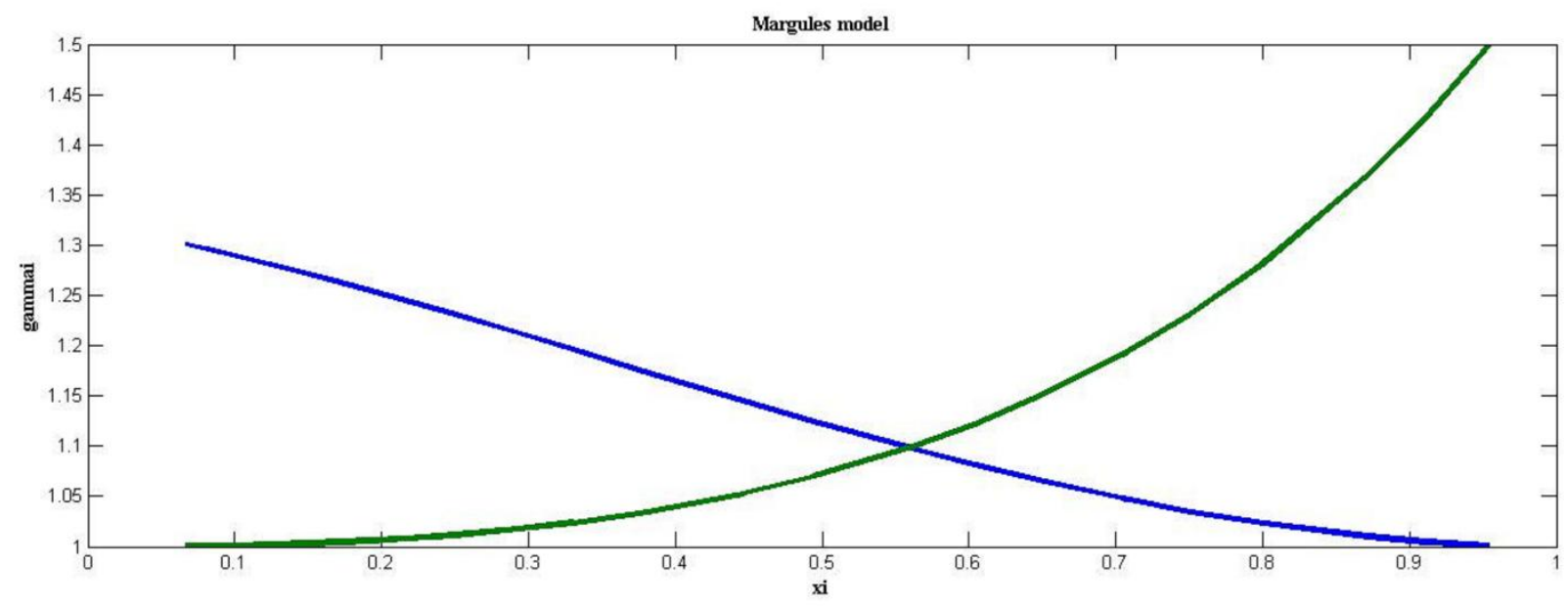

Figure 7- Plot of $\gamma_{1}$ and $\gamma_{2}$ vs $\mathrm{x}_{1}$ of Water(1)+Ethanoic acid(2) System at $101.25 \mathrm{kpa}$

\section{MATLAB CODE}

3.1 Code for determining activity coefficients of binary Methanol(1)+Water(2) System using various models. $\%$ $----\%$

\section{$\mathrm{P}=101.325$;}

$\mathrm{a} 1=7.19736 ; \mathrm{b} 1=1574.99 ; \mathrm{c} 1=-34.29$;

$\mathrm{a} 2=7.07404 ; \mathrm{b} 2=1657.46 ; \mathrm{c} 2=-46.13$;

$$
\text { \%-------------------VAN LAAR-------------------\% }
$$

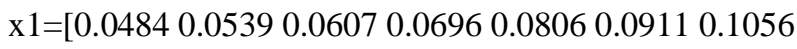

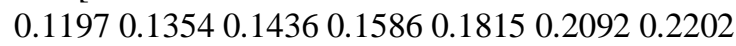

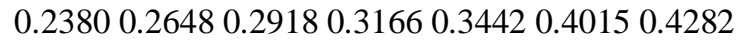

0.46440 .52400 .60910 .68990 .8213 ];

$\mathrm{y} 1=\left[\begin{array}{lllllll}0.2704 & 0.3009 & 0.3213 & 0.3492 & 0.3866 & 0.4126 & 0.4492\end{array}\right.$

0.47620 .48850 .50470 .53230 .56770 .59690 .6031

$\begin{array}{llllllll}0.6237 & 0.6519 & 0.6681 & 0.6923 & 0.7197 & 0.7431 & 0.7443\end{array}$

$\left.\begin{array}{llllll}0.7613 & 0.7860 & 0.8231 & 0.8633 & 0.9221\end{array}\right]$;

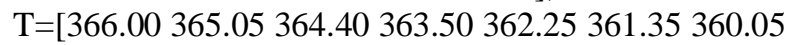

359.09358 .60358 .00356 .95355 .55354 .35354 .10

353.25352 .05351 .35350 .30349 .05347 .95347 .90

347.20346 .20344 .80343 .31341 .20 ];

Tsat $1=338.1 ;$ Tsat $2=373.16$;

$\mathrm{x} 2=1-\mathrm{x} 1$; y2=1-y1;

$\mathrm{p} 1=10 .^{\wedge}(\mathrm{a} 1-\mathrm{b} 1 . /(\mathrm{T}+\mathrm{c} 1))$;

$\mathrm{p} 2=10 .^{\wedge}(\mathrm{a} 2-\mathrm{b} 2 . /(\mathrm{T}+\mathrm{c} 2))$;

gamma1_exp=(y1.*P)./(p1.*x1);

gamma2_exp=(y2.*P)./(p2.*x2);

gamma1_calc $=$

$@(\mathrm{c}) \exp (\mathrm{c}(1) . *(((\mathrm{c}(2) . * \mathrm{x} 2) . /(\mathrm{c}(1) . * \mathrm{x} 1+\mathrm{c}(2) . * \mathrm{x} 2)) . \wedge 2))$; gamma2_calc $=$

$@(c) \exp \left(\mathrm{c}(2) . *\left(\left(\left(\mathrm{c}(1) \cdot{ }^{*} \mathrm{x} 1\right) \cdot /\left(\mathrm{c}(1) \cdot{ }^{*} \mathrm{x} 1+\mathrm{c}(2) .{ }^{*} \mathrm{x} 2\right)\right) \cdot{ }^{\wedge} 2\right)\right)$; ini_guess $=[1,1]$

$\mathrm{U}=@(\mathrm{c})\left(0.5^{*}(\operatorname{sum}((\right.$ gamma1_calc $(\mathrm{c})-$

gamma1_exp)+(gamma2_calc(c)-gamma2_exp)).^2))); display 'Vanlaar model activity coeffcients'

$[\mathrm{c}, \mathrm{fmin}]=$ fminsearch $(\mathrm{U}$, ini_guess $)$

gamma1_calcvalue $=\exp (\mathrm{c}(1) . *(((\mathrm{c}(2) . * \mathrm{x} 2) \cdot /(\mathrm{c}(1) . * \mathrm{x} 1+\mathrm{c}(2)$ $\left.\left.\left.{ }^{*} \mathrm{x} 2\right)\right) . \wedge 2\right)$ )

gamma2_calcvalue $=\exp \left(\mathrm{c}(2) \cdot *\left(\left(\mathrm{c}(1) \cdot{ }^{*} \mathrm{x} 1\right) . /\left(\mathrm{c}(1) \cdot{ }^{*} \mathrm{x} 1+\mathrm{c}(2)\right.\right.\right.$ .$\left.\left.\left.\left.^{*} \mathrm{x} 2\right)\right) \cdot{ }^{\wedge} 2\right)\right)$;

$\%$--------Graphs----------\%

subplot(3,3,1),plot(x1,T,'*',y1,T,'-*'),xlabel('x1(*),y1()'),ylabel('T'), title('Methanol-Water system'),subplot(3,3,2),plot(x1,gamma1_calcvalue,x2,gam ma2_calcvalue),xlabel('xi'),ylabel('gammai'),title('Van 
Laar model'), ,subplot(3,3,6),plot(x1,gamma1_exp,x1,gamma1_calcvalu e),xlabel('xi'),ylabel('gamma_exp \& gamma_calc'),title('Van Laar model');

\section{CONCLUSION}

The experimental data of three binary mixture i.e. methanol + water, methanol + ethanoic acid and water + ethanoic acid at $101.325 \mathrm{kpa}$ was correlated using the NRTL, Wilson, Margules, and van Laar equations. The vapor liquid equilibrium data of these binary mixture was available in literature. So activity coefficients of these systems were determined using MATLAB. It was shown that the deviations of NRTL, Wilson, Margules, and van Laar equations are reasonably small. The calculated bubble points accorded well with the experimental data. The results show that the calculated bubble point is well fitted by the models, which satisfy the need for the design and operation of separation process in chemical industry. Moreover, the method will provide theoretical guidance for the research of VLE data of strongly associating system of vapor and liquid phase in non-ideal behavior.

\section{REFERENCES}

[1] J.J.Ljunglin and H.C. van Ness, "Calculation of vapor-liquid equilibria from vapor pressure data," Chemical Engineering Science, vol.17, no. 7, pp.531-539, 1962.

[2] E.Hala, J.Pick, V.Fried, and O. Vilim, Vapor-Liquid Equilibrium, Pergamon, New York, NY, USA, 1958.

[3] J.Wisniak,A.Apelblat, and H.Segura, "Application of model-free computation techniques and limiting conditions for azeotropy. An additional assessment of experimental data," Chemical Engineering Science, vol. 52, no. 23, pp. 4393-4402, 1997.

[4] E. Lam, A. Mejia, H. Segura, J. Wisniak, and S. Loras, "A modelfree approach data treatment of vapor-liquid equilibrium data in ternary systems. . 1. Theory and numerical procedures," Industrial and Engineering Chemistry Research, vol. 40, no. 9, pp.2134-2148, 2001.

[5] E. Lam, A. Mejia, H. Segura, J. Wisniak, and S. Loras, “A modelfree approach data treatment of vapor-liquid equilibrium data in ternary systems.2.Application". Industrial and Engineering Chemistry Research, vol. 40, no. 9, pp.2149-2159, 2001.

[6] G.M. Wilson, "Vapor-liquid equilibrium. A new expression for the excess free energy of mixing," Journal of American Chemical Society, vol. 86, no. 2, pp.127-130, 1964.

[7] M.S. Margules, "On the composition of saturated vapors of mixtures," Akademie der Wissenschaften in Wien, MathematischNaturwissenschaftliche Klasse Abteilung 2, vol. 104, pp.1234$1239,1895$.

[8] H. Renon and J.M. Prausnitz, "Estimation of parameters for the NRTL equation for excess gibbs energies of strongly non-ideal liquid mixtures," Industrial \& Engineering Chemistry Process Design and Development, vol. 8, no. 3, pp. 413-419, 1969.

[9] J.J. Van Laar, "The vapor pressure of binary mixtures," Zeitschrift fur Physikalische Chemie, vol. 72, pp.723-751, 1910. 